\title{
Was die Schweiz von anderen Ländern lernen kann
}

\section{Yvonne Gilli}

Dr. med., Mitglied des Zentralvorstands der FMH und Departementsverantwortliche Digitalisierung und eHealth

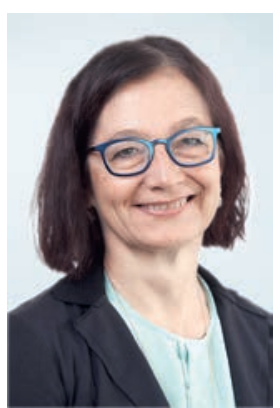

«Das Vergleichen ist das Ende des Glücks und der Anfang der Unzufriedenheit» ${ }^{1}$ oder mit anderen Worten: Vergleichen macht unglücklich. Trotzdem lesen wir immer gerne Berichte, die Lebensqualität, Bonität, Innovationsfähigkeit, menschliche Entwicklung oder die Schattenwirtschaft der Länder in Rankings vergleichen. Die Schweiz steht häufig gut da: Im Big-Mac-Index ${ }^{2}$ belegt die Schweiz den ersten Platz. Ob die Überbewertung des Schweizer Frankens nun gut oder schlecht ist und die Kaufkraft anhand von Sesambrötchen, Rinderhackfleisch, Schmelzkäse, Salat, Gewürzgurken, Zwiebeln und Saucen verglichen werden soll, bleibt dem Leser überlassen. Doch genau hier liegt das Problem von Ländervergleichen, insbesondere, wenn es um den Vergleich von Digitalisierungsstrategien im Gesundheitswesen geht. Erst kürzlich hat die Bertelsmann Stiftung in ihrem Bericht «SmartHealthSystems» die Digitalisierungsstrategien und den aktuellen Stand der Umsetzung von 14 EUMitgliedstaaten und drei OECD-Ländern (inklusive der Schweiz) verglichen. Aufgrund eines Fragebogens zum politischen Rechtsrahmen, der technischen Implementierung und der tatsächlichen Datennutzung wurde ein

\section{Der direkte digitale Datenaustausch zwischen Gesundheitsfachpersonen oder -institutionen birgt grosses Potenzial.}

sogenannter «Digital-Health-Index» für die teilnehmenden Staaten berechnet. Die Schweiz belegt dabei den viertletzten Platz, klassiert sich somit noch vor Frankreich, Deutschland und Polen. Im Vergleich zu den fortschrittlichsten Staaten wie Estland und Kanada ist auffallend, dass vor allem der Verbreitungsgrad von E-Rezepten, der Nutzungsgrad von elektronischen Patientenakten oder der elektronische Datenaustausch zwischen den Leistungserbringern gering ausfällt. ${ }^{3}$

Besonders der digitale Datenaustausch zwischen verschiedenen Gesundheitsfachpersonen oder -institutionen (Berichte, Laboraufträge, Verordnungen etc.) weist ein grosses Potenzial zur Effizienzsteigerung auf. Theoretisch könnten solche Zusatzdienste zur direkten Kommunikation zwischen Leistungserbringern ebenfalls die Infrastruktur des elektronischen Patientendossiers
EPD nutzen. Das Bundesgesetz zum EPD sieht jedoch vor, dass die EPD-Infrastruktur einzig für den primären Zweck des EPDs - zur zentralen Dokumentenablage verwendet werden darf. Hemmend für die Implementation von Zusatzdiensten kommt hinzu, dass die Entwick-

Man sollte sich auf politischer Ebene über nutzenstiftende Use-Cases unterhalten und entsprechende Rahmenbedingungen schaffen.

lung des elektronischen Patientendossiers komplexer ist, als zuerst angenommen wurde. Die Swisscom, welche die technische Plattform für die Stammgemeinschaft Axsana liefert, musste deshalb Anfang Jahr die Entwicklung von Zusatzdiensten zurückstellen, so dass die Infrastruktur für die zentrale Datenablage bis im Frühling 2020 rechtzeitig fertiggestellt werden kann. ${ }^{4}$ Wieso andere Länder im Bereich eHealth im Vergleich zur Schweiz fortschrittlicher sind, kann nicht unbedingt auf die Ausgangsbedingungen unseres Gesundheitssystems zurückgeführt werden. Beispielsweise ist das in der Studie der Bertelsmann Stiftung zweitplatzierte Kanada ebenfalls föderal organisiert und weist hinsichtlich des Gesundheitssystems zersplitterte Versorgungsstrukturen mit zentralen und regionalen Gesundheitsbehörden auf. Viel wichtiger ist nach Erfahrungen aus anderen Ländern eine starke strategische Einbindung aller Institutionen und Akteure und eine pragmatische, use-caseorientierte Vorgehensweise. Die Akzeptanz von digitalen Anwendungen durch Patientinnen und Patienten sowie Gesundheitsfachpersonen sollte dabei als strategische Aufgabe verstanden werden. Bedeutendes Mittel für die Akzeptanz der verschiedenen Akteure ist das systematische Co-Design mit Endanwendern statt einer hierarchisch geprägten Entwicklung Top-down.

Bei der Konzeption des EPDs wurden jedoch gerade diese «lessons learned» aus anderen Ländern nicht genügend berücksichtigt. Alternativ zu aktuellen politischen Debatten bezüglich einer Verpflichtung der ambulanten Ärzteschaft sollte man sich auch auf politischer Ebene vermehrt über konkrete nutzenstiftende Use Cases unterhalten und die dafür benötigten politischen Rahmenbedingungen schaffen. 